J. Clin. Chem. Clin. Biochem.

Vol. 23, 1985, pp. 765-775

\title{
Immunphänotypisierung von Lymphocyten-Subpopulationen mit monoklonalen Antikörpern im analytischen Durchflußcytometer: Evaluierung von Methodik und Referenzbereichen
}

\author{
Von D. Neumeier, Roswitha Pauls, Ingrid Wifling, A. Fateh-Moghadam und M. Knedel \\ Institut für Klinische Chemie (Direktor: Prof. Dr. M. Knedel), Klinikum Großhadern, Ludwig-Maximilians- \\ Universität München
}

(Eingegangen am 20. Mai/23. Juli 1985)

\begin{abstract}
Zusammenfassung: Moderne analytische Durchflußcytometer und monoklonale Antikörper gegen Oberflächendeterminanten von Lymphocyten haben die Immunphänotypisierung von Lymphocyten-Subpopulationen vereinfacht und beschleunigt. Wir untersuchten mononukleäre Zellen nach Dichtegradientenzentrifugation von EDTA-Vollblut. Die Intraassay-Präzision $(n=12)$ der Typisierung mit monoklonalen Antikörpern, die gegen T-Zellen (Leu 4), T-Helfer/Inducer-Zellen (Leu $3 \mathrm{a}, \mathrm{b}$ ), T-Suppressor/cytotoxische Zellen (Leu $2 \mathrm{a}$ ) und Zellen mit dem Phänotyp von NK-Zellen (Leu 7) gerichtet sind, schwankte zwischen 2,2 und 7,9\%. Untersuchungen mit Leu 2 a- und Leu 3 a, b-Antikörpern, die entweder mit Fluoresceinisothiocyanat (FITC) oder mit Phycoerythrin (PE) markiert waren, ergaben für beide Subpopulationen jeweils vergleichbare Ergebnisse. Simultane Doppelmarkierungen mit zwei verschiedenen Antikörpern, die mit kontrastierenden Fluorochromen konjugiert waren, zeigten gegenüber Einzelmarkierungen im Mittel gering höhere relative Anteile der T-Helfer/Inducer-Zellen (Leu 3a, b-FITC: $\mathrm{a}=0,97, \mathrm{~b}=3,18, \mathrm{r}=0.98$ ) und der T-Suppressor/ cytotoxischen Zellen (Leu $2 \mathrm{a}-\mathrm{PE}$ : $\mathrm{a}=0,99, \mathrm{~b}=2,22, \mathrm{r}=0,97$ ). Eine konstante Beziehung zur Klärung dieser statistisch signifikanten Unterschiede $(p \leqq 0,0002)$ fanden wir nicht. Die Untersuchung von 100 nicht kranken Kontrollpersonen ergab für den Anteil der Subpopulation folgende Referenzbereiche (Median, zentrales $95 \%$-Intervall): T-Zellen 0,75 (0,57-0,87); T-Helfer/Inducer-Zellen 0,47 (0,34-0,63); T-Suppressor/ cytotoxische Zellen $0,26(0,15-0,42)$; „NK“-Zellen $0,16(0,04-0,34)$. Die absoluten Zellzahlen $\left(10^{9} / 1\right)$ betragen: T-Zellen 1,300 (0,730-2,240); T-Helfer/Inducer-Zellen 0,820 (0,440-1,540); T-Suppressor/cytotoxische Zellen $0,450(0,190-0,915)$; „NK“-Zellen 0,300 (0,060-0,670).
\end{abstract}

Immunophenotyping of lymphocyte subsets by the use of monoclonal antibodies and analytical flow-cytometry: Evaluation of the method and reference values

Sümmary: Analytical flow cytometers which are easy to operate, and monoclonal antibodies specific for cell surface determinants of lymphocytes, offer a simple and rapid method for the identification of the immunological phenotype of lymphocyte subsets. We investigated mononuclear cells isolated from EDTA-blood bv density gradient centrifugation. The intraassay precision $(n=12)$ of the determination by means of monoclonal antibodies directed against T-cells (Leu 4), T-helper/inducer-cells (Leu 3a, b), T-suppressor/cytotoxic cells (Leu $2 \mathrm{a}$ ) and cells of the 'NK'-phenotype (Leu 7) varied between 2.2 and $7.9 \%$. Studies using the subset markers Leu $2 \mathrm{a}$ and Leu $3 \mathrm{a}, \mathrm{b}$ conjugated either to fluorescein isothiocyanate (FITC) or to phycoerythrin (PE) showed comparable results for both subsets. Dual immunofluorescent analysis using two different antibodies conjugated to contrasting fluorochromes resulted in slightly higher fractions of $T$-helper/inducercells (Leu 3 a, b-FITC: $\mathrm{a}=0.97, \mathrm{~b}=3.18, \mathrm{r}=0.98$ ) and T-suppressor/cytotoxic cells (Leu $2 \mathrm{a}-\mathrm{PE}: \mathrm{a}=0.99$, $\mathrm{b}=2.22, \mathrm{r}=0.97$ ) compared with single immunofluorescent analysis. We found no constant association to explain this statistically significant discrepancy ( $p \leqq 0.0002$ ). The investigation of 100 controls established 
the following reference values for the fraction of lymphocyte subsets (median, $95 \%$-interval): T-cells 0.75 (0.57-0.87), T-helper/inducer-cells 0.47 (0.34-0.63), T-suppressor/cytotoxic cells $0.26(0.15-0.42)$, 'NK' cells $0.16(0.04-0.34)$. The corresponding absolute numbers of cells are: T-cells $1.300(0.730-2.240), T$ helper/inducer-cells $0.820(0.440-1.540)$, T-suppressor/cytotoxic cells $0.450(0.190-0.915)$, 'NK'-cells 0.300 $(0.060-0.670)$.

\section{Einführung}

Die automatisierte Durchflußcytometrie hat heute eine Entwicklungsstufe erreicht, die eine breite Übernahme dieser Technologie aus den Forschungsbereichen in klinische Laboratorien erwarten läßt. Zwei sich gegenseitig ergänzende Entwicklungen sind dafür von ursächlicher Bedeutung:

Zunächst sind moderne, ausschließlich analytische Durchflußcytometer einfacher zu bedienen als bislang verfügbare Geräte, die vorwiegend für präparative Arbeiten konstruiert waren. Diese Durchflußcytometer ermöglichen eine schnelle, gleichzeitige und quantitative Analyse von bis zu 4 voneinander unabhängigen Kenngrößen einer in Suspension zu untersuchenden Zellpopulation. Diese technologische Entwicklung wurde vor allem durch Miniaturisierung der elektronischen Bauelemente und Fortschritte auf dem Gebiet der Fluorochrom-Chemie begünstigt. Daneben vermittelt die breite Verfügbarkeit monoklonaler Antikörper der Durchflußcytometrie gegenwärtig fortwährend neue Impulse. Als Reagenzien mit höchster Spezifität sind monoklonale Antikörper für weite Bereiche der Medizin, besonders in der Analytik und Identifikation komplexer Antigene von größter Bedeutung $(1-5)$.

Innerhalb des hämatopoetischen Systems des Menschen werden monoklonale Antikörper verwendet, um zelluläre Oberflächendeterminanten zu charakterisieren, die von normalen und malignen Zellen gebildet werden (6). Verschiedentlich ist es möglich, spezifische Zellfunktionen mit definierten Oberflächenmarkern in Beziehung zu setzen.

Die Quantifizierung von Lymphocyten-Subpopulationen hat klinische Bedeutung bei Erkrankungen, die durch Veränderungen im Immunsystem beeinflußt sind, so bei Transplantatabstoßung (7), primären und erworbenen Immunmangelzuständen $(8,9)$, rheumatoider Arthritis (10) oder multipler Sklerose (11). Die Bestimmung des immunologischen Phänotyps von Lymphocyten kann mit Hilfe der automatisierten Durchflußcytometrie oder manueller Mikroskopie nach Fluoreszenż- oder Immunogoldmarkierung (12) erfolgen.
Bislang sind für durchflußcytometrische Analysen kaum Daten zur Präzision und Reproduzierbarkeit der Methode außerhalb der speziellen Bedingungen von Forschungsbereichen mitgeteilt (13). Referenzbereiche sind meist für die Mikroskopie, häufig unter ungenügend definierten Bedingungen und innerhalb kleiner Kontrollgruppen, untersucht $(9,12,14-18)$. Daneben sind die Ergebnisse durch die Spezifität der monoklonalen Antikörper, die Probenvorbereitung und die Markierung beeinflußt $(14,16,19)$.

Es war deshalb Ziel dieser Arbeit, analytische Kriterien der Immunphänotypisierung von Lymphocyten mit einem automatischen Durchflußcytometer zu überprüfen und in einem ausreichend großen Kollektiv klinisch nicht kranker Personen die Verteilung der T-Zellen und „NK“-Zellen, sowie der T-Z̈ellenSubpopulationen $\mathrm{zu}$ untersuchen. Darüberhinaus sollte geklärt werden, inwieweit Untersuchungsergebnisse durch den zur Markierung des monoklonalen Antikörpers verwendeten Fluoreszenzfarbstoff und das methodische Vorgehen mit Einzel- oder simultaner Doppelmarkierung mit zwei verschiedenen monoklonalen Antikörpern beeinflußt werden.

\section{Material und Methoden}

Untersuchungskollektive

An 35 Patientenproben aus den Intensivpflegebereichen wurden die Vergleichsuntersuchungen der Fluorochrome und zur Einzel- und simultanen Doppelmarkierung von Oberflächendeterminanten durchgeführt.

Zur Abklärung der Referenzbereiche wurden unter $124 \mathrm{Be}-$ schäftigten des Krankenhauses, die sich nach ärztlicher Befragung nicht krank fühlten, 34 Männer (Alter: 21-54 Jahre, Median 34 Jahre) und 66 Frauen (Alter: 19-60 Jahre, Median 30 Jahre) ausgewählt, bei denen zusätzlich keine der bis zu 16 klinisch-chemischen und hämatologischen Kenngrößen der Basisdiagnostik eine Abweichung von den Referenzbereichen zeigte. Unter den hämatologischen Kenngrößen waren als Kriterien definiert:

Leukocyten, zwischen 3,8 und 8,5 (109/1), Anteil der Lymphocyten zwischen 0,19 und 0,41 (Haemalog 6010, Technicon, Bad Vilbel).

Die Probeentnahmen erfolgten in den Monaten Juli bis November 1984 in der Zeit zwischen 8.30 und 9:15 Uhr.

Zur Verlaufșkontrolle wurde bei einzẹlnen Personen dieses Kollektivs innerhalb eines Zeitraumes von 3-16 Wochen eine zweite Probenentnahme vorgenommien. 


\section{Probenmaterial}

Aus der Kubitalvene wurden $8 \mathrm{ml}$ Blut in Polystyrol-Injektionsspritzen (Braun, Melsungen) entnommen und sofort in je zwei $4 \mathrm{ml}$ EDTA-Gefäße (Greiner, Nürtingen) überführt.

\section{Monoklonale Antikörper}

Es wurden ausschließlich kommerziell erhältliche monoklonale Antikörper der Leu-Serie (Becton-Dickinson, Heidelberg) verwendet. Die T-Lymphocyten wurden typisiert mit an Fluoresceinisothiocyanat (FITC) konjugierten anti-Leu 4-Antikörpern. Die T-Helfer/Inducer- bzw. die T-Suppressor/cytotoxischen Zellen wurden über ihre Bindung an anti-Leu $3 \mathrm{a}$, b-Antikörper bzw. an anti-Leu 2a-Antikörper quantifiziert, wobei sowohl mit FITC als auch mit Phycoerythrin (PE) konjugierte monoklonale Antikörper verwendet wurden. FITC konjugierte antiLeu7-Antikörper wurden eingesetzt, um Zellen mit dem immunologischen Phänotyp von NK-Zellen zu quantifizieren. Die Spezifität dieser Antikörper ist mitgeteilt (20).

\section{Immunologische Phänotypisierung}

\section{Zellisolierung}

Als Standardmethode zur Isolierung der überwiegenden Zahl der Lymphocyten wurde eine Dichtegradienten-Trennung vorgenommen (21). Alle Präparationsschritte erfolgten in Polystyrolreagenzgefäßen (Greiner, Nürtingen).

Spätestens 20 Minuten nach Blutentnahme wurden das EDTABlut mit Hank's gepufferter Salzlösung (HBSS) (Serva, Heidelberg) im Verhältnis $1: 1$ verdünnt. Jeweils $6 \mathrm{ml}$ dieser Mischung wurden vorsichtig über $3 \mathrm{ml}$ Ficoll-Hypaque (Pharmacia, Heidelberg) geschichtet und bei Raumtemperatur für $30 \mathrm{~min}$ mit $400 \mathrm{~g}$ zentrifugiert. Nach Zentrifugation wurde der Überstand abgesaugt. Die zweite Schicht, die vorwiegend mononukleäre Zellen enthält, wurde mit einer Pasteur-Pipette aufgenommen und zweimal mit etwa $8 \mathrm{ml}$ HBSS gewaschen $(100 \mathrm{~g}, 10 \mathrm{~min}$, $4^{\circ} \mathrm{C}$ ). Nach dem Waschen wurden die Zellen erneut in etwa $1 \mathrm{ml}$ HBSS resuspendiert, um die Zellzahl auf etwa $10^{7} / \mathrm{ml}$ einzustellen. Ab diesem Schritt wurde die weitere Probenvorbereitung bis zur Auswertung lichtgeschützt im Eisbad bei $4^{\circ} \mathrm{C}$ durchgeführt.

\section{Antikörperbindung}

Im Eisbad wurden $50 \mu \mathrm{l}$ der isolierten Zellen in ein $3 \mathrm{ml}$ Analysenröhrchen (Becton Dickinson, Heidelberg) überführt und mit $5 \mu l$ des konjugierten monoklonalen Antikörpers vermischt. Bei Ansätzen in simultaner Doppelmarkierungs-Technik wurden in jedes Analysenröhrchen zusätzlich $5 \mu$ l eines zweiten monoklonalen Antikörpers, der mit dem kontrastierenden Fluorochrom konjugiert und gegen eine zweite Oberflächen-Determinante gerichtet war, zugegeben. Nach Inkubation (30 min) wurden die Zellen mit $2 \mathrm{ml}$ gekühlter, Phosphat-gepufferter $\mathrm{NaCl}$-Lösung $(0,15 \mathrm{~mol} / 1, \mathrm{pH} 7,4)$ gewaschen $\left(200 \mathrm{~g}, 5 \mathrm{~min}, 4^{\circ} \mathrm{C}\right)$. Als Kontrolle für eine unspezifisçè Bindung des monoklonalen Anti.körpers (Maus) über die Fc-Région wurde jeweils ein $50 \mu \mathrm{l}$ Aliquot der isolierten Zellen mitgeführt, dem $5 \mu$ l eines FITCmarkierten Maus-IgG-Serums zugegeben wurden. Alle Markierungen erfolgten in Doppelansätzen.

\section{Analyse im Durchflußcytometer}

Die Zellpräparationen wurden in einem automatisierten Durchflußcytometer (FACS-Analyser, Becton-Dickinson, Heidelberg) analysiert und on-line über den Rechner, Consort $30^{\circ}$ (BectonDickinson, Heidelberg) ausgewertet. Das Durchflußcytometer ermöglicht die gleichzeitige, quantitative Erfassung von bis zu vier voneinander unabhängigen Kenngrößen.
Die Funktion des Analysengerätes beruht im Prinzip darauf, $\mathrm{da} B$ die Zellen der Suspension über ein Kapillarsystem im Zentrum eines umhüllenden Pufferstroms hydrodynamisch fokussiert und damit in einer perlschnurähnlichen Formation durch eine konische Meßöffnung mit $100 \mu \mathrm{m}$ Innendurchmesser geführt werden. Von jeder durchtretenden Zelle (Fließgeschwindigkeit 200 bis 300 Zellen pro Sekunde) werden nach Anregung im fokussierten Lichtstrahl einer Quecksilberlampe bis zu zwei kontrastierende Fluoreszenzsignale und ein $90^{\circ}$ Streulicht-Signal (proportional dem Ausmaß der Heterogenität oder der Granulierung der Zelle) erfaßt. In einem weiteren Kanal wird über eine Impedanzmessung die Zellgröße bestimmt. Nach Analog-Digital-Wandlung werden diese Signale zellbezogen in den Kernspeicher eines Oszillographen übernommen. Auf dem Bildschirm können diese Signale von bis zu 1000 Zellen einzeln oder in beliebiger Kombination paarweise dargestellt werden.

Gleichzeitig können die Kenngrößen zellbezogen auf ein Speichermedium des Rechners für eine spätere rechnerunterstützte Auswertung übernommen werden. Da über das Streulichtsignal und die Zellgröße Lymphocyten, Monocyten und Granulocyten charakterisiert werden (Abb. 1 a), können über elektronische Analysenfenster diese Zellen selektiv nach der Intensität ihrer Fluoreszenzsignale, entsprechend der Oberflächendichte der markierten Antigendeterminante, untersucht werden. Ausgehend von den nicht markierten Kontrollproben, kann in beiden Fluoreszenzkanälen für jede Zellpopulation eine Intensitätsgrenze der Autofluoreszenz festgelegt werden. In den markierten Proben sind so innerhalb der interessierenden Zellpopulation unmarkierte Zellen von markierten Zellen zu unterscheiden (Abb. 1 b). In einer biparametrischen Darstellung beider Fluoreszenzintensitäten können doppelt markierte Zellen erfaßt werden (Abb. 2). Über die Auswerteprogramme werden die absolute Zellzahl und die relativen Anteile der markierten Zellen sowohl innerhalb des jeweiligen Analysenfensters als auch in Bezug auf die Gesamtzahl der untersuchten Zellen ausgegeben. In jeder Probe werden in der Regel 5000 bis 10000 Zellen untersucht. Aus der Zahl der Leukocyten $\left(10^{9} / 1\right)$ in EDTA-Blut und dem Anteil der Lymphocyten im Differentialblutbild der Kontrollperson wurde die absolute Zellzahl $\left(10^{9} / 1\right)$ der Lymphocyten-Subpopulation berechnet.

\section{Statistik}

Zur Berechnung der Referenzbereiche der Lymphocyten-Subpopulationen wurden jeweils Mittelwerte aus Doppelbestimmungen herangezogen. Die Prüfung auf eine Geschlechts- und Altersabhängigkeit der Referenzbereiche erfolgte mit dem UTest nach Mann-Whitney. Im Rahmen der methodischen Vergleichsuntersuchungen wurde für Wertepaare eine standardisierte Hauptkomponenten-Analyse (22) und der WilcoxonPaardifferenzentest durchgeführt.

\section{Ergebnisse}

Durch die Signale der Impedanz-Messung und des $90^{\circ}$-Streulichtes ist die Lymphocytenpopulation gegenüber anderen mononukleären Zellen charakterisiert (Abb. 1 a). Damit kann die so definierte Population selektiv nach der Intensität ihrer Fluoreszenzsignale ausgewertet werden (Abb. 1 b, c). Bei Doppelmarkierungen mit FITC- und PE-konjugierten Antikörpern werden die Fluoreszenzsignale in beiden Auswertekanälen ohne störende Interferenz des kontrastierenden Fluorochroms erfaßt (Abb. 2). Das 


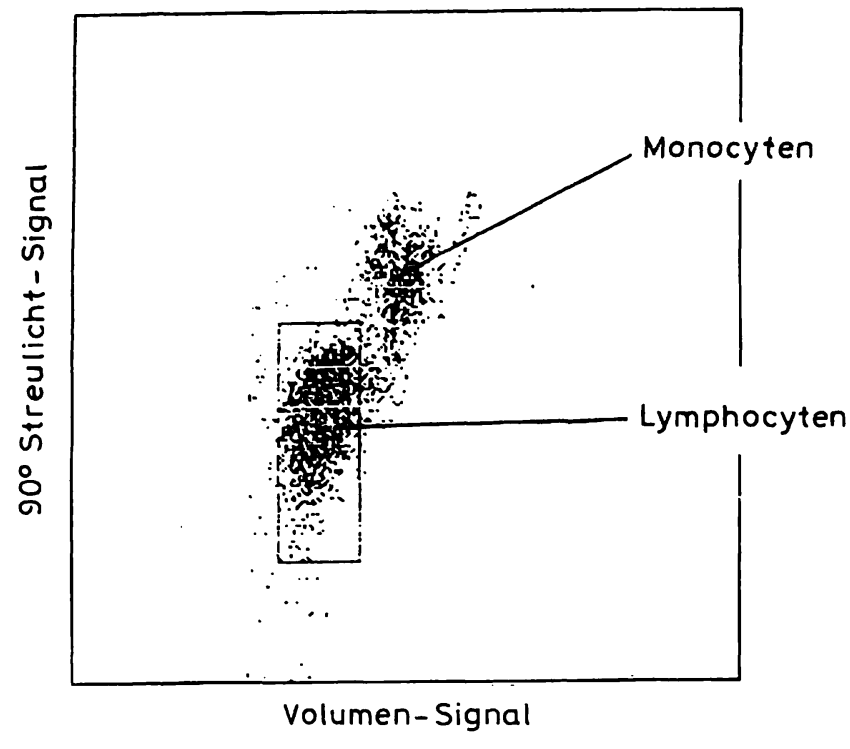

Abb. 1 a. Volumen-Streulicht-Diagramm (x-Achse: Volumensignal, $y$-Achse: $90^{\circ}$-Streulichtsignal) einer Zellsuspension nach Dichtegradienten-Zentrifugation aus EDTA-Vollblut einer nicht kranken Kontrollperson. Das eingezeichnete Rechteck entspricht einem elektronischen Fenster zur selektiven Auswertung dieser Zellpopulation (Lymphocyten).

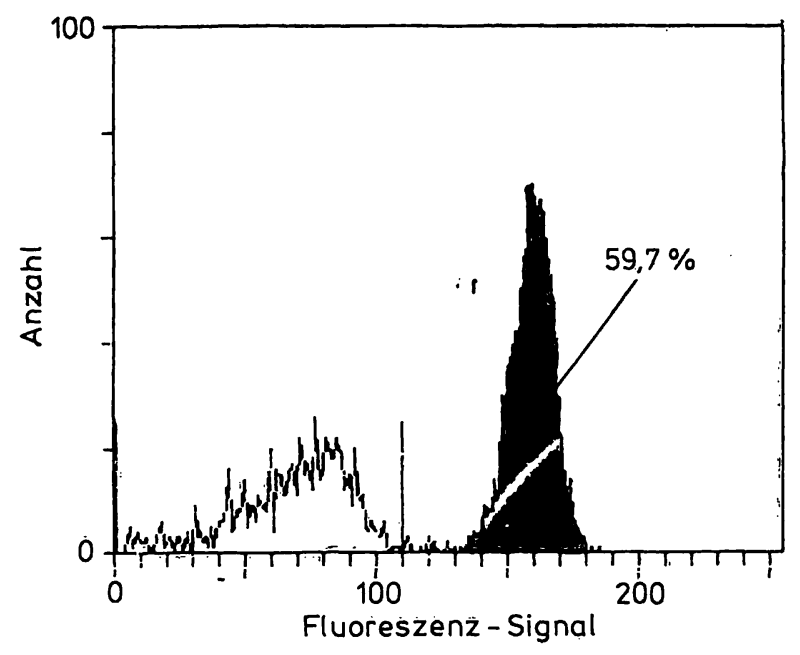

Abb. 1 b. Typische Verteilung des Fluoreszenzsignals (FITC) einer mit anti=Leu 4 (T-Zellen) markierten Probe einer nicht kranken Kontrollperson. Dargestellt sind nur Zellen innerhalb des Lymphocyten-Auswertefensters (definiert durch Volumensignal und $90^{\circ}$-Streulichtsignal). Die mit Antikörpern markierten Zellen sind gegenüber den nicht markierten Zellen in der Intensität des emittierten Fluoreszenzsignals zu unterscheiden. Die Markierung bei der Skalenteilung ,110' entspricht der oberen Intensitätsgrenze der Autofluoreszenz einer unbehandelten Präparation derselben Kontrollperson. Der Anteil der markierten Zellen innerhalb des elektronischen Auswertefensters beträgt bei dieser Probe $59,7 \%$.

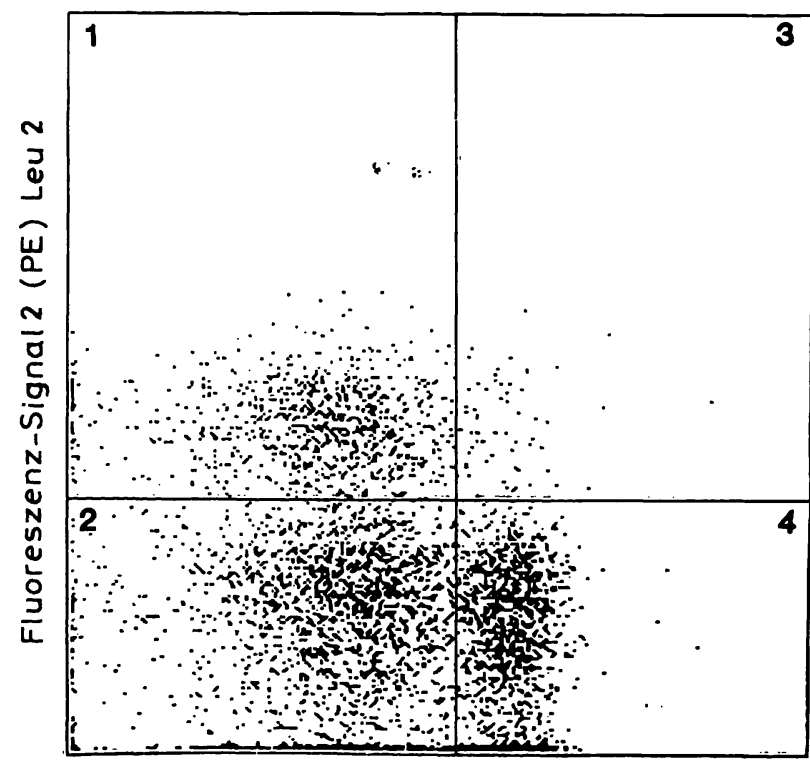

Flụoreșzenz-Signal 1 (FITC) Leu 3

Abb. 2. Diagramm der Fluoreszenzsignale der Lymphocytenpopulation einer nicht kranken Kontrollperson nach Doppelmarkierung mit anti-Leu $3 \mathrm{a}$, b (Fluoresceinisothiocyanat, FITC) und anti-Leu 2 a (Phycoerytherin, PE).

Sektor 1, Zellen mit dem Phänotyp Leu $3 \mathrm{a}, \mathrm{b}^{-}$, Leu $2 \mathrm{a}^{+}$; Sektor 2, Zellen mit dem Phänotyp Leu $3 \mathrm{a}, \mathrm{b}^{-}$, Leu $2 \mathrm{a}^{+}$; Sektor 3 , Zellen mit dem Phänotyp Leu $3 \mathrm{a}, \mathrm{b}^{+}$, Leu $2 \mathrm{a}^{+}$; Sektor 4 , Zellen mit dem Phänotyp Leu $3 \mathrm{a}^{+} \mathrm{b}^{+}$, Leu $2 \mathrm{a}^{-}$. 
Zur weiteren Abklärung der Präzision der Bestimmung wurden die Wertepaare aus Doppelansätzen herangezogen, die durch dieselbe Assistentin an verschiedenen Tagen analysiert wurden. Der Variationskoeffizient wurde nach der Formel $\sqrt{\left(\Sigma \mathrm{d}^{2}\right) / 2 \mathrm{n}}$ berechnet, wobei d die Differenz der Doppelwerte in Prozent und $\mathrm{n}$ die Zahl der Bestimmungen angibt. Für die prozentualen Anteile der Subpopulationen liegen diese Variationskoeffizienten bei Einzelmarkierungen zwischen 2,2 und $7,9 \%$ (Tab. 2).

\section{Einzel- und simultane Doppelmarkierung der Zellen}

In den Vergleichsuntersuchungen zum Einfluß des zur Konjugation an den monoklonalen Antikörper verwendeten Fluoreszenzfarbstoffes und zur Methodik der Immunphänotypisierung mit Einzel- oder simultaner Doppelmarkierung ergeben sich Korrelationskoeffizienten zwischen 0,93 und 0,98. Die Steigungen der Hauptkomponentengeraden betragen zwischen 0,96 und 0,99 . Die y-Achsenabschnitte liegen zwischen -0,54 und 3,36 (die Angaben beziehen sich auf den relativen Anteil der T-Zellen-Subpopulation) (Tab. 3, Abb. $3 \mathrm{a}, \mathrm{b}$ ).

Tab. 3. Ergebnisse der standardisierten HauptkomponentenAnalyse für Wertepaare nach Einfachmarkierungen mit monoklonalen Antikörpern, die mit FITC oder PE konjugiert sind bzw. nach simultaner Doppelmarkierung mit zwei verschiedenen monoklonalen Antikörpern, die mit kontrastierenden Fluorochromen konjugiert sind. Angegeben sind $\mathrm{r}=$ Regressionskoeffizient, Steigung der Hauptkomponentengerade und Intercept $=$ Achsenabschnitt.

\begin{tabular}{|c|c|c|c|c|}
\hline & & \multicolumn{3}{|c|}{$\begin{array}{l}\text { Hauptkomponenten- } \\
\text { Analyse }\end{array}$} \\
\hline & & $r$ & $\begin{array}{l}\text { Stei- } \\
\text { gung }\end{array}$ & $\begin{array}{l}\text { Inter- } \\
\text { cept }\end{array}$ \\
\hline \multirow{5}{*}{$\begin{array}{l}\text { Leu } \\
3 a, b \\
N=35\end{array}$} & $\begin{array}{l}\text { Einzelmarkierungen } \\
\text { FITC/PE }\end{array}$ & 0,94 & 0,96 & 0,59 \\
\hline & & & & \\
\hline & FITC & & & \\
\hline & $\begin{array}{l}\text { Einzel-/ } \\
\text { Doppelmarkierungen }\end{array}$ & 0,98 & 0,97 & 3,18 \\
\hline & $\begin{array}{l}\text { PE-Einzelmarkierung } \\
\text { FITC-Doppelmarkierung }\end{array}$ & 0,93 & 0,98 & 3,36 \\
\hline \multirow{4}{*}{$\begin{array}{l}\text { Leu } \\
2 \mathrm{a} \\
\mathrm{N}=3\end{array}$} & $\begin{array}{l}\text { Einzelmarkierung } \\
\text { FITC/PE }\end{array}$ & 0,96 & 0,99 & $-0,54$ \\
\hline & & & & \\
\hline & $\begin{array}{l}\text { PE } \\
\text { Einzel-/ } \\
\text { Doppelmarkierung }\end{array}$ & 0,97 & 0,99 & 2,22 \\
\hline & $\begin{array}{l}\text { FITC-Einzelmarkierung } \\
\text { PE-Doppelmarkierung }\end{array}$ & 0,93 & 0,96 & 1,70 \\
\hline
\end{tabular}

Tab. 2. Präzision aus Doppelbestimmungen der relativen Anteile von Lymphocyten-Subpopulationen nach Immunphänotypisierung.

\begin{tabular}{lllll}
\hline Antikörper & $\mathrm{N}$ & $\begin{array}{l}\text { VK } \\
(\%)\end{array}$ & $\mathrm{s}$ & $\overline{\mathrm{x}}$ \\
\hline Leu 4 & 78 & 2,20 & 0,0160 & 0,733 \\
Leu 2a & 82 & 5,38 & 0,0138 & 0,257 \\
Leu 3a, b & 69 & 4,10 & 0,0192 & 0,470 \\
Leu 7 & 72 & 7,87 & 0,0138 & 0,175 \\
\hline
\end{tabular}
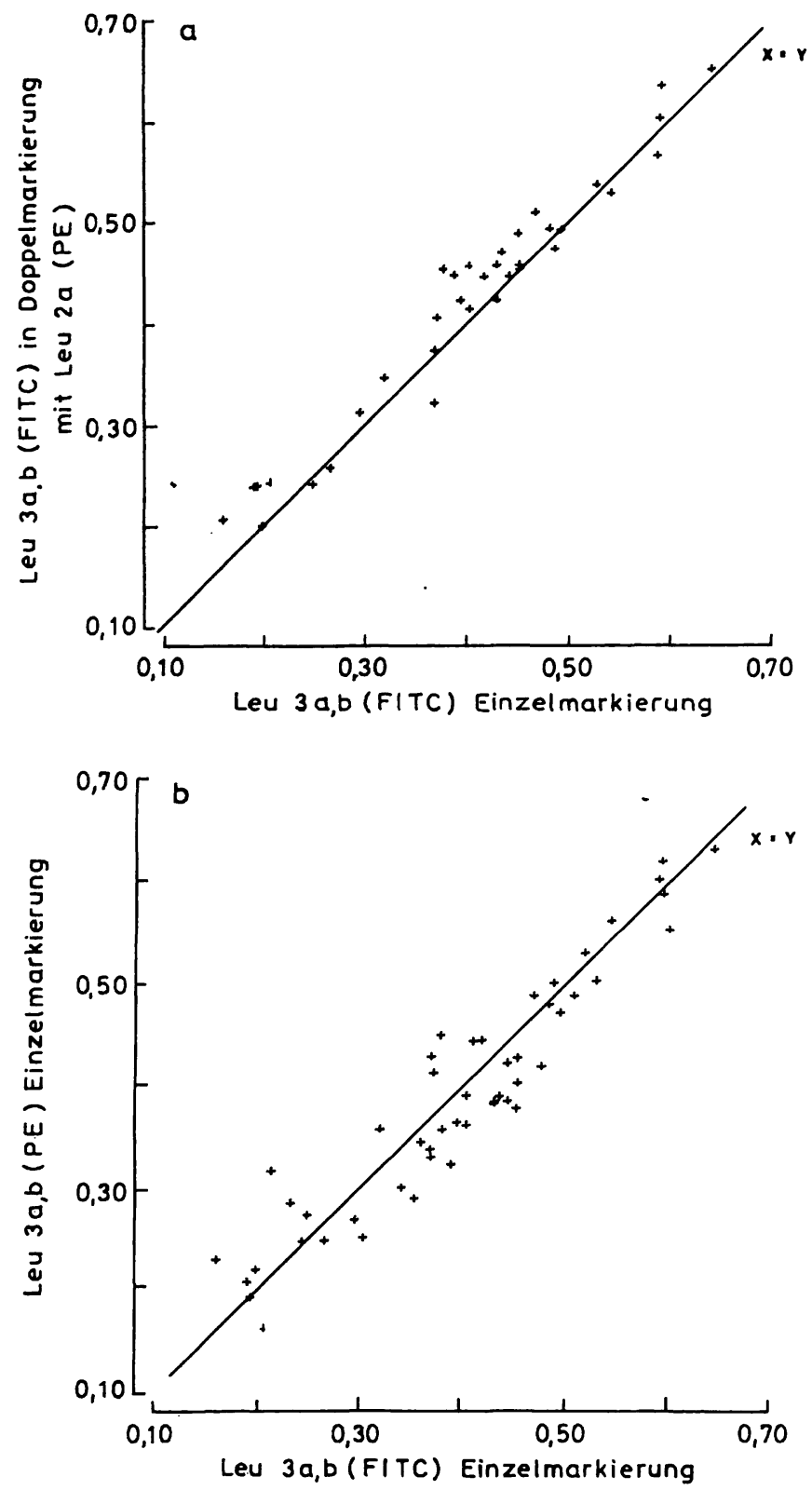

Abb. 3. Vergleiche der relativen Anteile der T-Zellen-Subpopulationen in Einzelmarkierung bzw. gleichzeitiger Zugabe von zwei verschiedenen monoklonalen Antikörpern (Leu $3 \mathrm{a}, \mathrm{b}$ und Leu $2 \mathrm{a}$ ), die mit kontrastierenden Fluorochromen konjugiert sind (Doppelmarkierung) und Einzelmarkierungen mit monoklonalen Antikörpern, die mit verschiedenen Fluorochromen konjugiert sind. a) anti-Leu 3a,b markierte Zellen: FITCkonjugierter Antikörper in Einzelmarkierung bzw. simultaner Doppelmarkierung mit anti-Leu 2 a (PE); b) anti-Leu 3 a,b markierte Zellen: Einzelmarkierung mit einem FITC- bzw. PE-konjugierten Antikörper. 
Tab. 4. Referenzberciche von Lymphocyten-Subpopulationen im peripheren Blut bei nicht kranken Kontrollpersonen nach durchflußcytometrischer Bestimmung des immunologischen Phänotyps mit monoklonalen Antikörpern. Die Angaben in $10^{9} / 1$ ergeben sich aus dem Anteil der Subpopulation sowie der Gesamtzahl der Leukocyten und dem Anteil der Lymphocyten im peripheren Blut der Kontrollperson.

\begin{tabular}{|c|c|c|c|c|c|c|}
\hline \multirow[t]{2}{*}{ Zellen } & \multirow[t]{2}{*}{ Antikörper } & \multicolumn{2}{|l|}{ Anteil } & \multicolumn{2}{|c|}{ Gesamtzahl $\left(10^{9} / 1\right)$} & \multirow[t]{2}{*}{$\mathbf{N}$} \\
\hline & & Median & $\begin{array}{l}95 \% \\
\text { Intervall }\end{array}$ & Median & $\begin{array}{l}95 \% \\
\text { Intervall }\end{array}$ & \\
\hline $\begin{array}{l}\text { T-Zellen } \\
\text { T-Helfer } \\
\text { T-Suppressor } \\
\text { „NK"-Zcllen }\end{array}$ & $\begin{array}{l}\text { Leu } 4 \\
\text { Leu } 3 a, b \\
\text { Leu } 2 a \\
\text { Leu } 7\end{array}$ & $\begin{array}{l}0,75 \\
0,47 \\
0,26 \\
0,16\end{array}$ & $\begin{array}{l}0,57-0,87 \\
0,34-0,63 \\
0,15-0,42 \\
0,04-0,34\end{array}$ & $\begin{array}{l}1,300 \\
0,820 \\
0,450 \\
0,300\end{array}$ & $\begin{array}{l}0,730-2,240 \\
0,440-1,540 \\
0,190-0,915 \\
0,060-0,670\end{array}$ & $\begin{array}{r}100 \\
100 \\
100 \\
93\end{array}$ \\
\hline
\end{tabular}
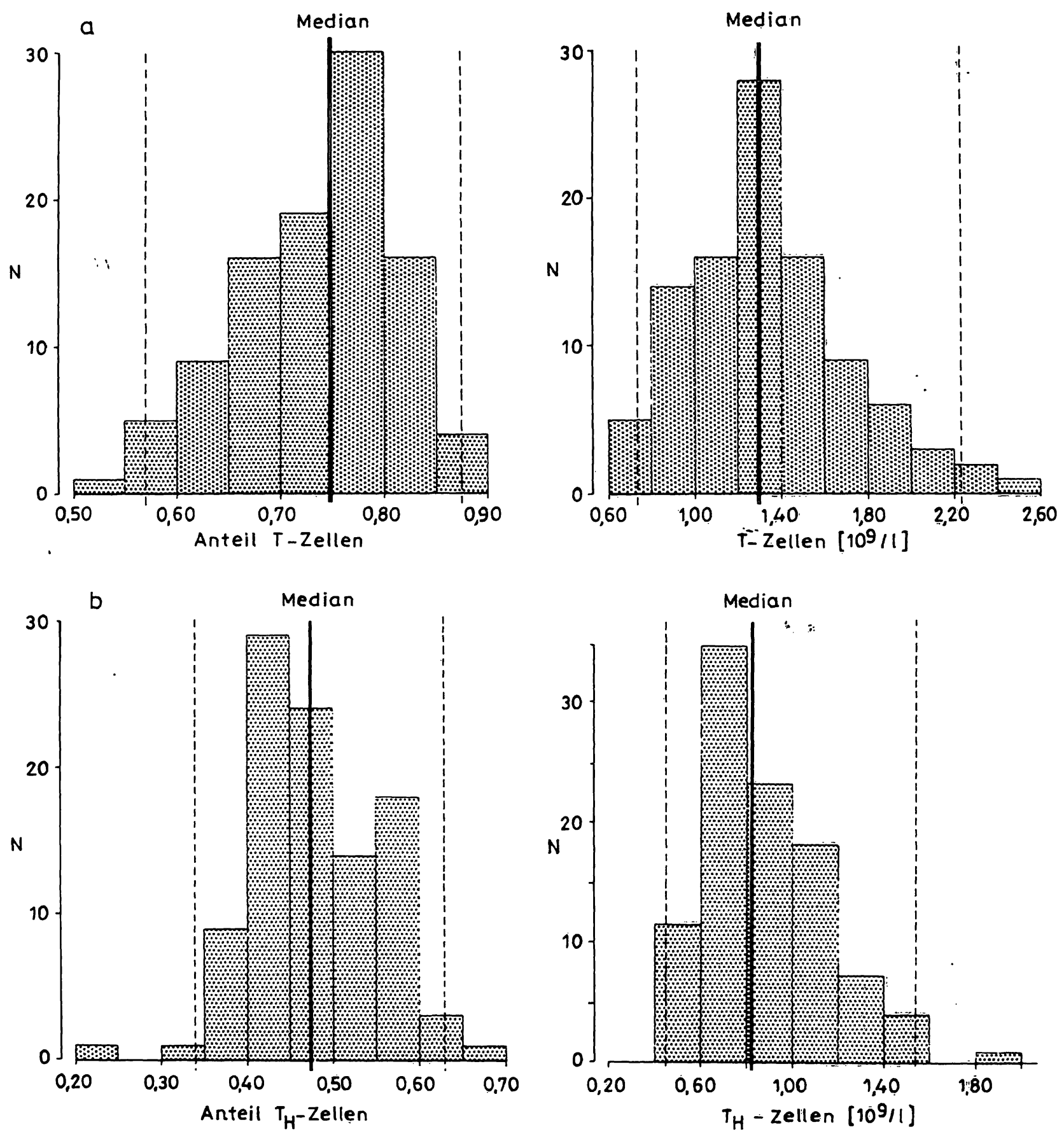

Abb. 4. Häufigkeitsverteilung von Lymphocyten-Subpopulationen im peripheren Blut bèi nícht kranken Kontrollpersonen.

a) T-Lymphocyten (Leu 4), b) T-Helfer-Zellen (Leu 3a, b), c) T-Suppressor-Zellen (Leu 2 a), d) Zellen mit dem immunologischen Phänotyp von NK-Zellen (Leu 7). Jeweils links Anteil der Population innerhalb der im LymphocytenAuswertefenster analysierten Zellen, jeweils rechts die Zellzahl $\left(10^{9} / 1\right)$, die sich aus Gesamtzahl der Leukocyten $\left(10^{9} / 1\right)$ im peripheren Blut und dem Anteil der Lymphocyten im Differential-Blutbild errechnet. Die gestrichelten Linien entsprechen der 5\%- und 95\%-Perzentile. 
Werden im Vergleich Einzel- und simultane Doppelmarkierung durchgeführt und wird zur Konjugation an den monoklonalen Antikörper jeweils der gleiche Fluoreszenzfarbstoff verwendet, so finden sich in den Doppelmarkierungen sowohl für die T-Helfer/Inducer- als auch für die T-Suppressor/cytotoxischen Zellen im Mittel höhere relative Anteile. Diese Unterschiede sind im Wilcoxon-Paardifferenzentest signifikant ( $\mathrm{p} \leqq 0,0002)$ (Abb. $3 \mathrm{a})$.

Werden die Ergebnisse aus Einzelmarkierungen miteinander verglichen, wenn die monoklonalen Antikörper entweder mit FITC oder PE konjugiert sind, so sind die Unterschiede weder für die anti-Leu $2 \mathrm{a}$ noch die anti-Leu $3 \mathrm{a}, \mathrm{b}$ markierten Populationen si-
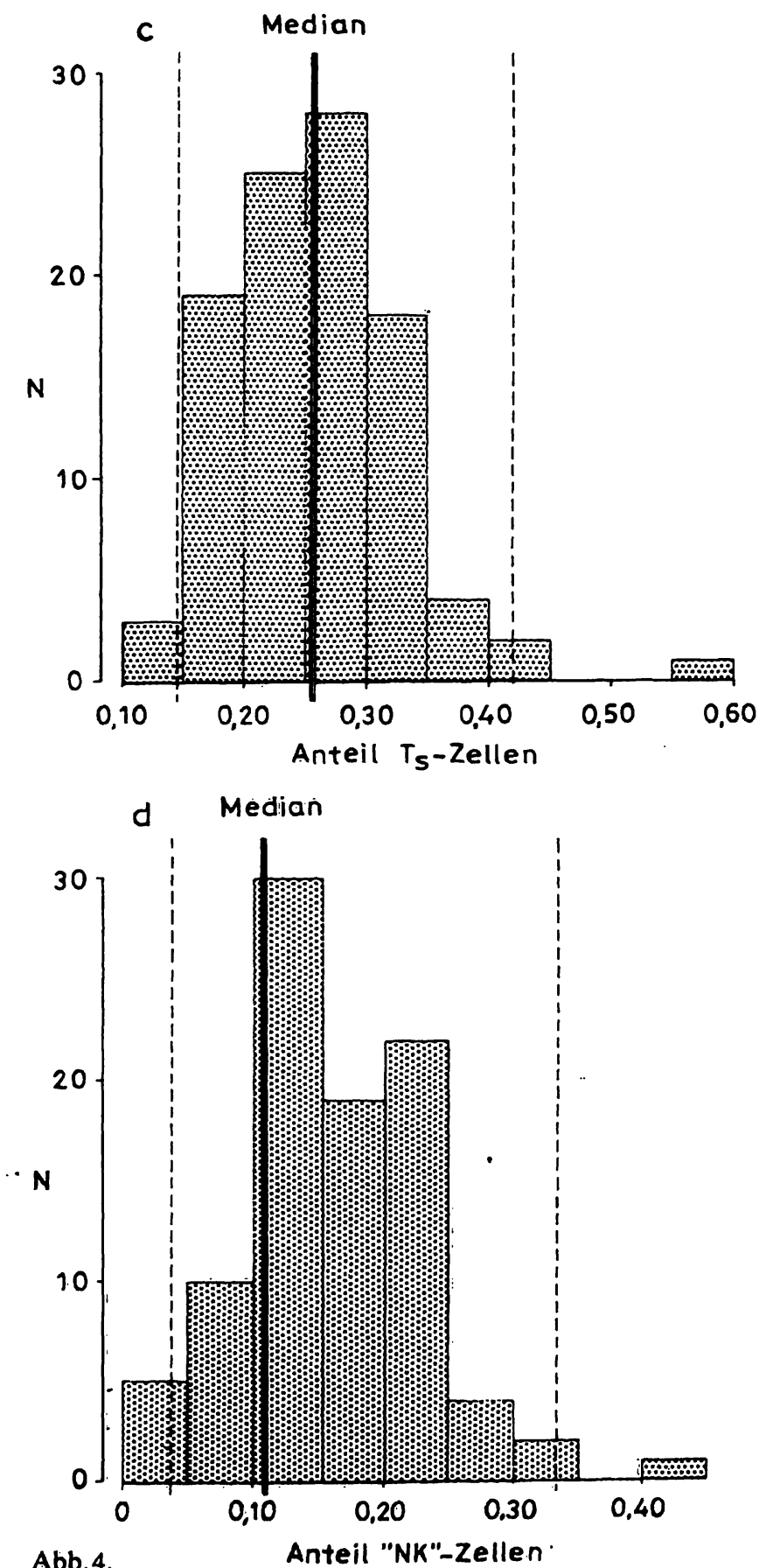

gnifikant. Es sei aber darauf hingewiesen, daß bei einzelnen Patienten die Ergebnisse um bis zu 8 Relativprozent differieren können (Abb. 3 b).

\section{Referenzbereiche}

Bei klinisch nicht kranken Kontrollpersonen kann der relative Anteil der typisierten Lymphocyten-Subpopulationen innerhalb weiter Bereiche schwanken. Ausgeprägte Unterschiede zeigen sich ebenso, wenn aus der Zahl der Leukocyten $\left(10^{9} / 1\right)$ in EDTA-Blut und dem Anteil der Lymphocyten im Differentialblutbild der Kontrollperson die absolute Zellzahl $\left(10^{9} / 1\right)$ der Subpopulation errechnet wird (Tab. 4, Abb. $4 \mathrm{a}-\mathrm{d}$ ). Bei keiner der Subpopulationen besteht
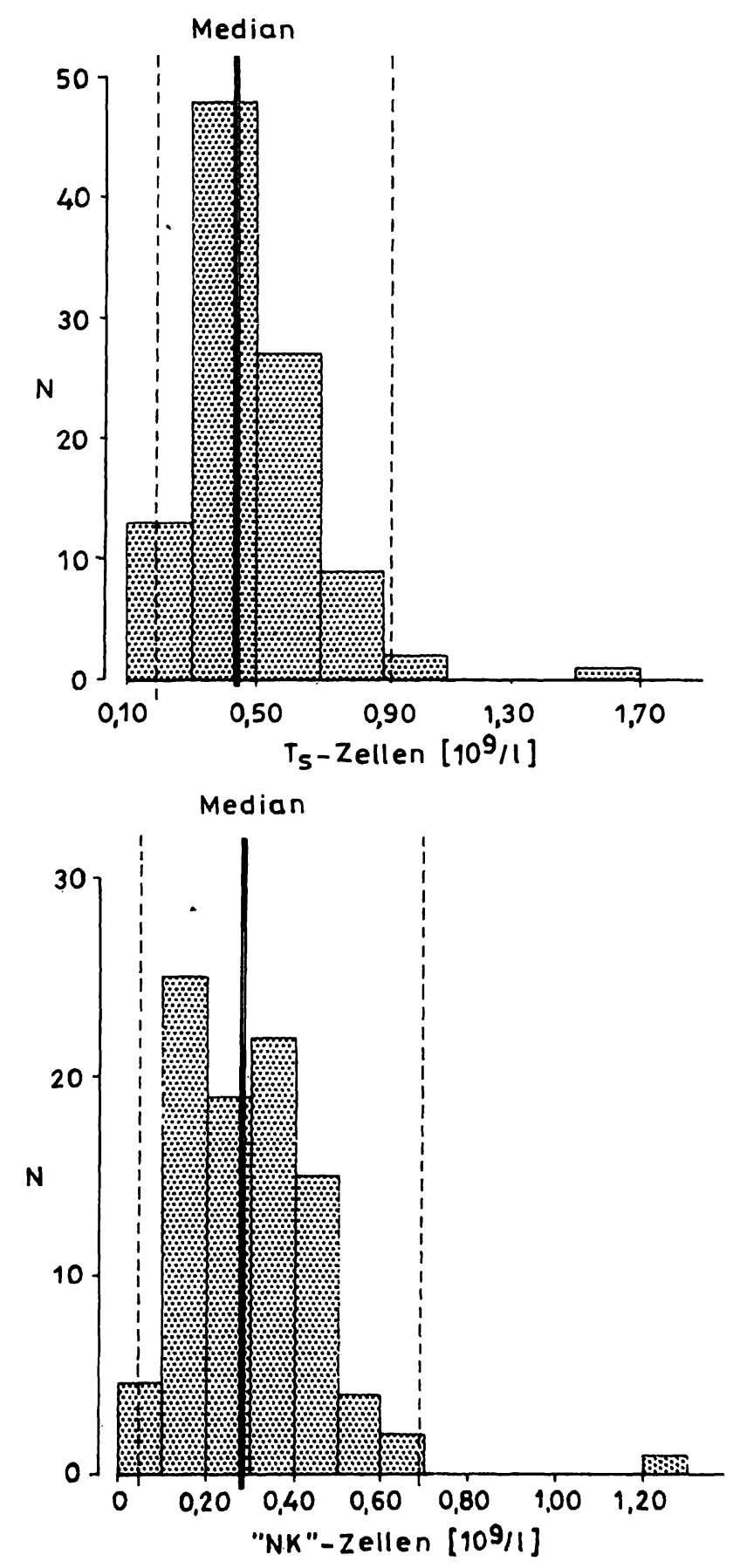
eine Abhängigkeit zwischen der Höhe des relativen Anteils und der absoluten Zellzahl (10 $/ 1)$ (Abb. $5 \mathrm{a}-\mathrm{d})$.

Hingewiesen sei auf Befunde einer weiblichen Kontrollperson, bei der relativer Anteil und absolute Zellzahl der T-Suppressor/cytotoxischen Zellen und der mit anti-Leu 7 typisierten „NK“-Zellen weit außerhalb der zentralen 95\%-Intervalle des Kollektivs liegen. Eine Kontrolluntersuchung nach 12 Wochen bestätigte diese Befunde. Die erneute ärztliche Untersuchung und Kontrolle klinisch-chemischer und häma- tologischer Kenngrößen ergab keinen pathologischen Befund. Eine Abhängigkeit des Referenzintervalls von Geschlecht oder Alter (Gruppierung nach dem Altersmedian) der Kontrollpersonen ergibt sich für keine der Subpopulationen (Test nach Mann-Whitney).

Für die klinisch wichtige Hilfsgröße des Quotienten aus den T-Helfer/Inducer- und T-Suppressor/cytotoxischen Zellen liegt der Referenzbereich zwischen 1,00 und 3,65, bei einem Median von 1,87. Im erwähnten Einzelfall findet sich ein Quotient von 0,41 (Abb. 6).
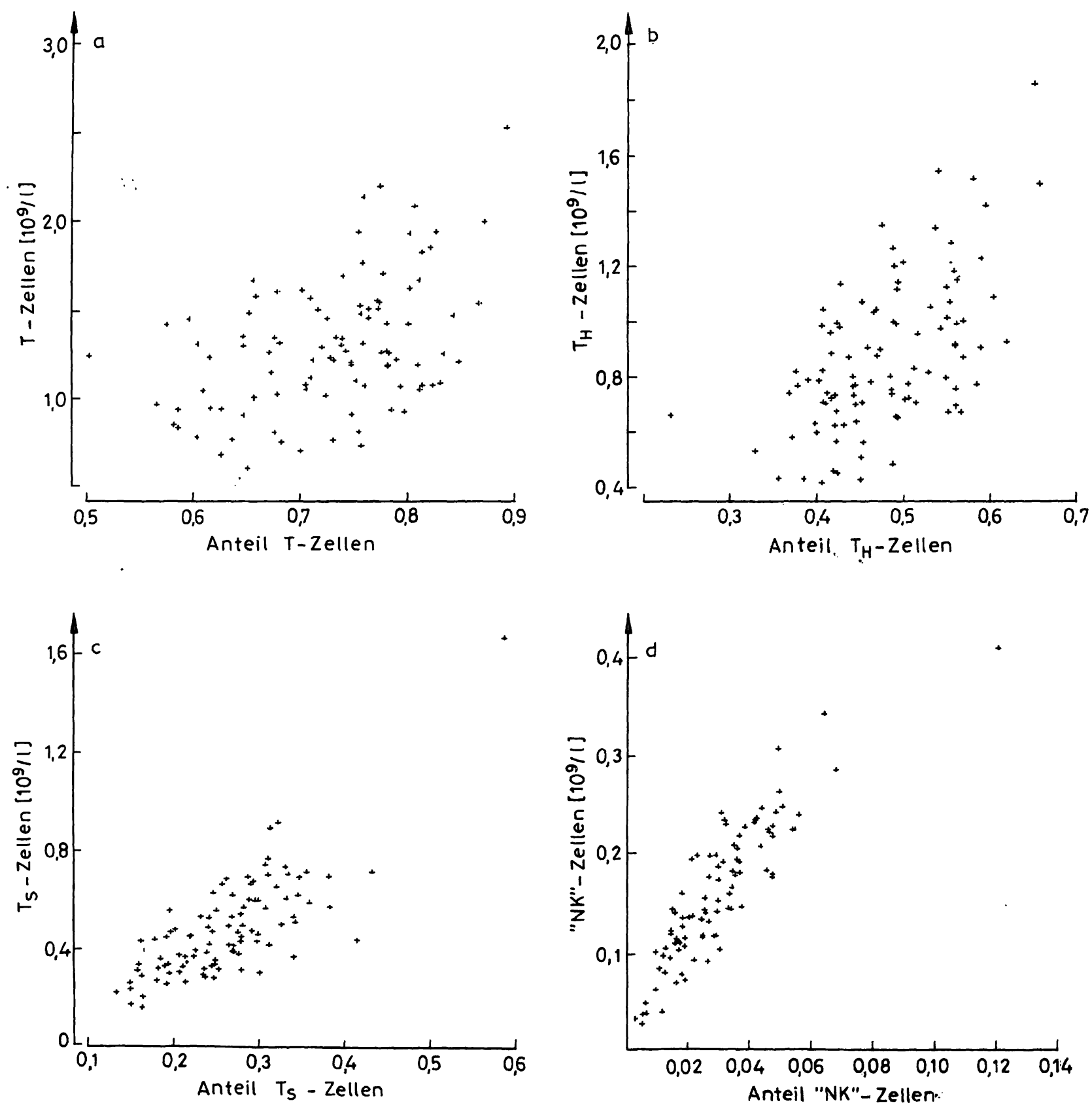

Abb. 5. Lymphocyten-Subpopulationen im peripheren Blut nicht kranker Kontrollpersonen. Vergleich des relativen Anteils und der Zellzahl $\left(10^{9} / 1\right)$ der Subpopulationen (s. Abb. 4).

a) T-Lymphocyten (Leu 4), b) T-Helfer-Zellen (Leu 3a, b'), c) T-Suppressor-Zellen (Leu 2a), d) Zellen mit dem immunologischen Phänotyp von NK-Zellen (Leu 7). 


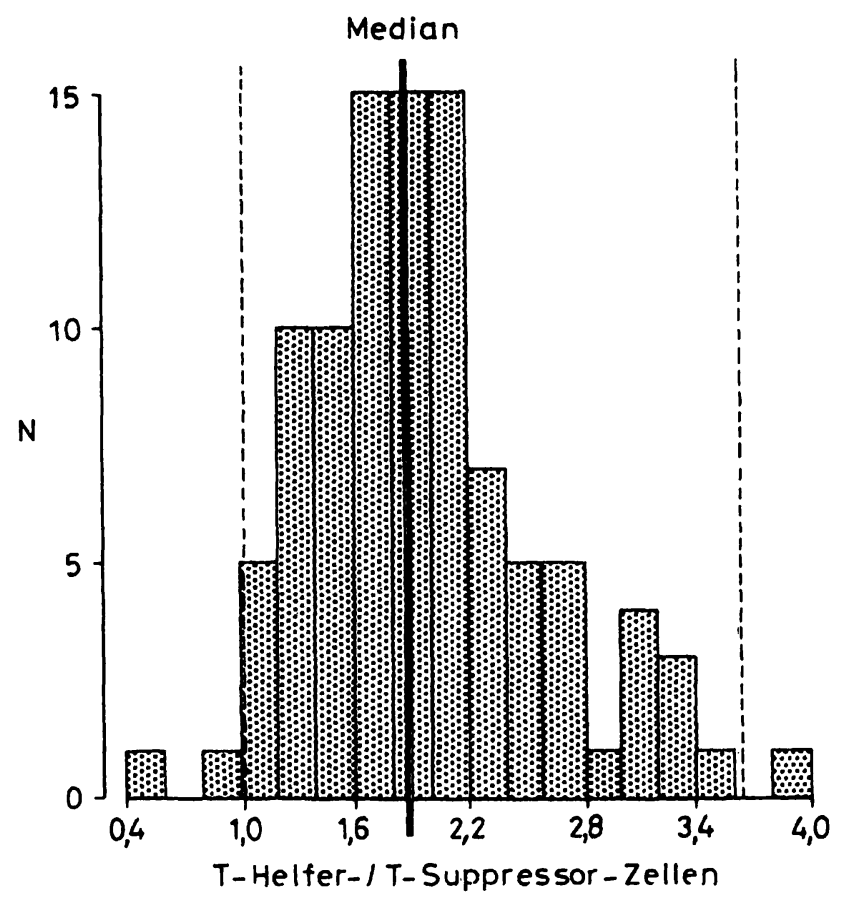

Abb. 6. Häufigkeitsverteilung der Quotienten aus T-Helferund T-Suppressor-Zellen bei nicht kranken Kontrollpersonen $(n=100)$. Die gestrichelten Linien entsprechen der 5\%-und $95 \%$-Perzentile.

In einer zweiten Probennahme wurden 13 Kontrollpersonen nach einem Intervall von 3 bis 16 Wochen (Median: 66 Tage) untersucht. Die Ergebnisse zeigen, daß sich die absoluten Zellzahlen der T-Helfer/Inducer- und T-Suppressor/cytotoxischen Zellen bei diesen Personen, bei denen sich in keinem Fall ein Hinweis auf eine intercurrent abgelaufene Erkrankung fand, in der Regel nur geringfügig verändern. Im WilcoxonPaardifferenzen-Test ergibt sich kein Hinweis auf signifikante Untersç hiede der Wertepaare.

\section{Diskussion}

Unter den Methoden zur Erfassung und Kontrolle der zellulären Immunregulation steht die Immunphänotypisierung im analytischen Durchflußcytometer oder durch Fluoreszenzmikroskopie neben der bekannten Zelldifferenzierung in Funktionstesten $(23-25)$ oder der Quantifizierung von löslichen, zellspezifischen Antigenen im Immunoassay (26) oder durch Prôteinmapping (27).

Funktionsteste fanden außerhalb von Forschungslaboratorien kaum Verbreitung, da die Zugabe von Lymphocyten mit Helfer- oder Suppressorfunktion in Kulturen zur Quantifizierung funktioneller Effekte, wie Antikörperproduktion oder DNA-
Synthese, zeitaufwendig, sehr methodenabhängig und an die Interaktion verschiedener Zellpopulationen gebunden ist (25). Darüberhinaus werden verschiedene Subpopulationen durch die Analyse ihrer funktionellen Eigenschaft nur ungenügend charakterisiert (28, 29). Mit der Immunphänotypisierung wird dagegen eine einfachere und zum Teil weitergehende Charakterisierung der Zellen möglich.

Wichtige Vorteile der automatisierten Durchflußcytometrie gegenüber der manuellen Fluoreszenzmikroskopie sind die große Zahl objektiv analysierter Zellen (in einer typischen Analyse etwa 5000 Zellen innerhalb des Lymphocyten-Auswertefensters) sowie die verbesserten Möglichkeiten einer simultanen Doppelmarkierung und einer Quantifizierung der Antigendichte auf einzelnen Zellen. Gleichzeitig wird Zeit und Probenvolumen eingespart. Allerdings ist die fehlende Information zur Zellmorphologie und -architektur für bestimmte Fragestellungen ein Nachteil (20).

Besonders für die klinische Routinediagnostik ergeben sich durch automatisierte analytische Durchflußcytometer, die eine gleichzeitige zellbezogene Fluoreszenzanalyse von 2 Oberflächendeterminanten ermöglichen und die ohne aufwendige Laser-Lichtquelle arbeiten, neue und interessante Möglichkeiten. Doppelmarkierungen gestatten zeitsparend eine simultane Analyse der beiden wichtigen Subpopulationen der T-Zellen. Ebenso kann bei spezieller Fragestellung mit einem zweiten monoklonalen Antikörper eine weitergehende Differenzierung der durch den ersten Antikörper definierten Zellpopulation (28) oder eine Abgrenzung gegenüber unspezifischen Bindungen (30) erfolgen.

Eine vorausgehende Isolierung der Lymphocyten ist zur Charakterisierung und Immunphänotypisierung im Durchflußcytometer nicht zwingend erforderlich. Direkte Bestimmungen in lysierten Vollblutproben sind möglich $(16,19)$. Wir bevorzugen jedoch die Abtrennung mononukleärer Zellen im Dichtegradienten, da im Volumen-Streulicht-Diagramm die Charakterisierung der Lymphocyten gegenüber den übrigen Zellen und Debris eindeutiger ist und da im lysierten Vollblut bei verschiedenen Erkrankungen eine Kontamination der im Lymphocyten-Auswertefenster zu analysierenden Zellen mit Granulocyten und Monocyten bekannt ist (31). Darüberhinaus scheint der Lyse-Puffer bei einzelnen Proben die Reaktivität der zellulären Oberflächendeterminanten zu beeinflussen (16). Dagegen führen Zellverluste im Dichtegradienten auch nach unseren Erfahrungen nicht zu klinisch relevanten Veränderungen des Musters mononukleärer Zellen im venösen Blut (14). 
Die Untersuchungen zur Methodik zeigen, daß eine gute Intraassay-Präzision mit Variationskoeffizienten unter 7\% auch außerhalb von Entwicklungslaboratorien (13) gegeben ist. Wiederholungsuntersuchungen an aufeinander folgenden Tagen führten wir nicht durch, da einzelne Oberflächendeterminanten, insbesondere auf den T-Helfer-Zellen, offensichtlich wenig stabil sind (32). Für die Interassay-Präzision dürften aber vergleichbare Variationskoeffizienten anzunehmen sein, wenn die Geräteeinstellung mit Fluorochrom markierten Latexpartikeln täglich überprüft wird.

In Doppelmarkierungen finden sich gegenüber Einzelmarkierungen gering höhere relative Anteile der T-Helfer/Inducer- und T-Suppressor/cytotoxischen Zellen. Für klinische Fragestellungen sind diese statistisch signifikanten Unterschiede bislang ohne wesentliche Bedeutung. Die Ursache dieser Veränderung, die bei FITC- und PE-konjugierten Antikörpern zu beobachten ist und für die sich auch in der Literatur Hinweise finden (16), ist nicht abgeklärt. Eine Interferenz bei der Erfassung der Fluoreszenzsignale durch den kontrastierenden Farbstoff und eine veränderte Autofluoreszenz wurden in jedem Ansatz überprüft und sind auszuschließen. Auch ein von der Durchflußrate am Cytometer abhängiger Effekt liegt nicht vor. $\mathrm{Zu}$ diskutieren ist dagegen eine veränderte Affinität der monoklonalen Antikörper bei simultaner Doppelmarkierung. In Einzelmarkierungen beeinflußt der Fluoreszenzfarbstoff die Ergebnisse nicht signifikant.

Für die klinische Beurteilung von Untersuchungsergebnissen ist der Vergleich mit Ergebnissen aus Kontroll- oder Referenzkollektiven von entscheidender Bedeutung. In vielen Untersuchungen, die zu verschiedenen Erkrankungen durchgeführt wurden $(9,12,15,17,18)$, finden sich Angaben zur Verteilung der Lymphocyten-Subpopulationen in Kontrollkollektiven. Infolge methodischer Unterschiede sowie kleiner und häufig ungenügend charakterisierter Kollektive differieren diese Angaben und sind nur bedingt übertragbar.

In dem ärztlich untersuchten und nach der Wertlage klinisch-chemischer und hämatologischer KenngröBen der Basisdiagnostik von uns ausgewählten Kollektiv können infolge der zeitlich standardisierten Abnahmebedingungen Veränderungen durch Einflußgrößen wie Tagesrhythmik (33-37), saisonale Schwankungen (38) und körperliche Belastung (39) ebenso wie durch akute oder weitverbreitete chronische Erkrankungen weitgehend ausgeschlossen werden. Eine Normalverteilung der Ergebnisse, wie sie von anderen Autoren angenommen wurde $(16,40)$, ist weder für die relativen Anteile noch für die absoluten Zellzahlen der Lymphocyten-Subpopulationen gegeben. Zur Definition der Referenzbereiche ist deshalb das zentrale $95 \%$-Intervall heranzuziehen.

Vergleichbare Referenzbereiche für die relativen Anteile der T-Zellen-Subpopulationen werden von Miller et al. (15) beschrieben, die eine kleine Kontrollgruppe mit korrespondierender Analysenmethode (Dichtegradienten-Separation, Durchflußcytometrie mit präparativen Geräten) untersuchten. Ähniliche Ergebnisse wurden kürzlich auch mit einer simultanen Doppelmarkierungstechnik in lysierten Vollblutproben mitgeteilt (16). Demgegenüber werden nach Auszählung der Zellen im Fluoreszenzmikroskop niedrigere relative Referenzbereichsgrenzen angegeben (14). Dies kann auf Probleme bei der Klassifizierung von Zellen mit mäßiger Fluoreszenzintensität und unterschiedliche Bezugsgrößen (alle mononukleären Zellen bzw. nur Lymphocyten) zurückgeführt werden.

Unsere Untersuchungen ergaben keinen Hinweis auf eine Geschlechtsabhängigkeit der relativen Referenzbereiche der Lymphocyten-Subpopulationen. Dies bestätigt frühere an einem kleineren Kontrollkollektiv erhobene Befunde (31).

Für klinische Fragestellungen, insbesondere im Rahmen der Verlaufsbeurteilung, ist die Angabe der absoluten Zellzahl $\left(10^{9} / 1\right)$ einer Subpopulation der Relativangabe vorzuziehen, da Veränderungen einer Population bei geänderter Leukocyten- oder Lymphocytenzahl besser erkannt werdèn $(14,40)$. Bemerkenswert in diesem Zusammenhang ist das vom übrigen Kollektiv abweichende Muster der Lymphocyten-Subpopulationen bei einer weiblichen Kontrollperson. Eine Ursache für diese Veränderung mit den hohen Anteilen an T-Suppressor/cytotoxischen Zellen und „NK“Zellen fanden wir nicht. Möglicherweise wird mit den bislang eingesetzten monoklonalen Antikörpern der immunologische Phänotyp nur unvollständig charakterisiert, so daß bei einzelnen Personen keine sichere Aussage zum funktionellen Verhalten der so typisierten Zellpopulationen möglich ist (41).

Die Untersuchung zeigt, da $\beta$ wichtige Marker des immunologischen Phänotyps von Lymphocyten im analytischen Durchflußcytometer einfach und sehr schnell identifiziert und quantifiziert werden können. Eine gute, für den klinischen Gebrauch ausreichende analytische Präzision ist gegeben. Simultane Doppelmarkierungen von zwei Oberflächenmarkern mit monoklonalen Antikörpern, die mit kontrastierenden Fluorochromen konjugiert sind, können die Ergebnisse gegenüber Einzelmarkierjungen geringfügig ver- 
ändern. Bei klinisch nicht kranken Kontrollpersonen können Anteil und Zellzahl der Lymphocyten-Subpopulationen innerhalb relativ weiter Bereiche schwanken.

Die Bestimmung des immunologischen Phänotyps von Lymphocyten-Subpopulationen wird infolge dieser verbesserten und vereinfachten Analytik und des größer werdenden Spektrums verfügbarer monoklonaler Antikörper eine weite Verbreitung finden. Die gegenwärtig an vielen Orten und bei verschiedenen Erkrankungen durchgeführten Untersuchungen werden die klinische Bedeutung weiter abklären. Zur Einsparung von Probenmaterial und Zeit sowie zur besseren Beurteilung der Befunde erscheint es uns zweckmäßig, diese Untersuchungen gemeinsam mit den übrigen Kenngrößen der hämatologischen und immunologischen Basisdiagnostik durchzuführen. Abhängig von der klinischen Fragestellung ist eine parallele oder stufenweise Analytik möglich.

\section{Literatur}

1. Damjanow, I. \& Knowles, B. B. (1983) Lab. Invest. 48, $510-525$.

2. Sinkovics, J. G. \& Dreesman, G. R. (1983) Rev. Infect. Dis. 5, $9-34$.

3. Sevier, E. D., David, G. S., Martinis, J., Desmond, W. J., Bartholomew, R. M. \& Wang, R. (1981) Clin. Chem. 27, 1797- 1806.

4. Diamond, B. A., Yelton, D. E. \& Scharff, M. D. (1981) N. Engl. J. Med. 304, 1344-1348.

5. Seiler, F. R., Gronski, P., Kurrle, R., Lüber, G., Harthus, H.-P., Ax, W., Bosslet, K. \& Schwick, H.-G. (1985) Angew. Chemie 97, 141-163.

6. Thiel, E. (1984) Rec. Res. Cancer Res. 93, 102-158.

7. Colvin, R. B. (1984) Ann. N. Y. Cad. Sci. 428, 5-13.

8. Pinching, A. J. (1984) Clin. Exp. Immunol. 56, 1-13.

9. Buckley, R. H., Gard, S., Schiff, R. I. \& Sampson, H. A. (1983) In: Primary immunodeficiency diseases (Wedgwood, R. J., Rosen, F. S. \& Paul, N. W., eds.), A. R. Liss, Inc., Nèw York, pp. 187-191.

10. Bertouch, J. V., Roberts-Thomson, P. J., Brooks, P. M. \& Bradley, J. (1984) J. Rheumatol. 11, 754-759.

11. Ellison, G. W., Visscher, B. R., Graves, M. C. \& Fahey, J. L. (1984) Ann. Intern. Med. 101, 514-526.

12. Toamszewski, J. E., Zimijewski, C. M. \& Rosenberg, J. S. (1984) Transplantation 38, $591-594$.

13. Ip, S. H., Rittershaus, C. W., Healey, K. W., Struzziero, C. C., Hoffman, R. A. \& Hansen, P. W. (1982) Clin. Chem. $28,1905-1909$.

14. Goff, L. K., Habeșhaw, J. A., Rose, M. L., Gracie, J. A. \& Gregòry, W. (1985) J. Clin. Pathol. 38, 54-59.

15. Miller, K. B., Elta, G. H., Rudders, R. A. \& Kaplan, M. M. (1984) Ann. Intern. Med. 100, 385-387.

16. Thornthwaite, J. T., Seckinger, D., Sugarbaker, E. V., Rosenthal, P. K. \& Vazqueż, D. A. (1984) Am. J. Clin. Pathol. $82,48-56$.

17. Cooksey, G., Robins, R. A. \& Blamey, R. W. (1984) Br. J. Surg. 71, 874-877.

18. Fujita, T., Matsui, T., Nakao, Y. \& Watanabe, S. (1984) Min. Elect. Metab. 10, 375-378.

19. Hoffman, R. A., Kung, P. C., Hansẹn, W. P. \& Goldstein, G. (1980) Proc. Nat. Acad. Sci. (USA) 77, 4914-4917.

20. Lovett, E. J., III., Schnitzer, B., Keren, D. F., Flint, A., Hudson, J. L. \& McClatchey, K. D. (1984) Lab. Invest. 50, $115-140$.

21. Böyum, A. (1968) Scand. J. Clin. Lab. Invest. 21 (Suppl. 97), $77-89$.

22. Feldmann, U., Schneider, B., Klinkers, H. \& Haeckel, R. (1981) J. Clin. Chem. Clin. Biochem. 19, 121-137.

23. Kersey, J. H. \& Gajl-Peczalska, K. J. (1975) Am. J. Pathol. $81,446-457$.

24. Gupta, S. \& Good, R. A. (1980) Sem. Haematol. 17, 1-28.

25. Knight, S. C. (1982) J. Immunol. Methods S0, R51-R63.

26. Folks, T. M. \& Sell, K. W. (1983) J. Immunol. Methods $57,43-50$.

27. Lester, E. P., Lemkin, P. F. \& Lipkin, L. E. (1984) Ann. N. Y. Acad. Sci. 428, 159-172.

28. Romain, P. L. \& Schlossman, S. F. (1984) J. Clin. Invest. $74,1559-1565$.

29. Morimoto, C., Reinherz, E: L., Distaso, J. A., Steinberg, A. D. \& Schlossman, S. F. (1984) J. Clin. Invest. 73, $689-700$.

30. Ryan, D. H., Mitchell, S. J., Hennessey, L. A., Bauer, K. D., Horan, P. K. \& Cohen, H. J. (1984) J. Immunol. Meth. 74, 115-129.

31. Iwatani, Y., Amino, N., Mori, H., Asari, S., Ina, K., Ennyu, K. \& Miyai, K. (1982) J. Immunol. Methods 54, $31-42$.

32. Weiblein, B. J., Debell, K. \& Valeri, C. R. (1983) N. Engl. J. Med. 309, 763.

33. Levi, F., Cannon, C., Blum, J. P., Reinberg, A. \& Mathe, G. (1983) Lancet $I I, 462-463$.

34. Levi, F. A., Canon, C., Blum, J.-P., Mechkouri, M., Reinberg, A. \& Mathe, G. (1985) J. Immunol. 134, 217-222.

35. Ritchie, A. W. S., Oswald, I. \& Micklem, H. S. (1983) Br. Med. J. 286, 1773-1775.

36. Bertouch, J. V., Roberts-Thompson, P. J. \& Bradley, J. (1983) Br. Med. J. 286, $1171-1172$.

37. Knapp, M. S. \& Pownall, R. (1984) Br. Med. J. 298, $1328-1330$.

38. Abo, T., Miller, C. A., Cloud, G. A. \& Blach, C. M. (1985) J. Clin. Immunol. 5, 13-20.

39. Edwards, A.J., Bacon, T. H., Elms, C. A., Verardi, R., Felder, M. \& Knight, S. C. (1984) Clin. Exp. Immunol. 58, 420-427.

40. Burmester, G. R. \& Kalden, J. R. (1983) Lab. Med. 7, $225-230$.

41. Kimber, I. (1985) Med. Lab. Sci. 42, 60-77.

PD Dr. Dieter Neumeier

Institut für Klinische Chemie

Klinikum Großhadern

Ludwig-Maximilians-Universität

Postfach 701260

D-8000 München 70 
